\title{
PLANTA DIDÁTICA PARA CONTROLE DE VELOCIDADE E DE POSIÇÃO ANGULAR DE UM MOTOR ELÉTRICO DE CORRENTE CONTÍNUA: USO DO MÉTODO PID DISCRETIZADO
}

Peter Franklin Ribeiro de Souza - peter.souza@ifmg.edu.br Universidade Federal de Viçosa

Av. Peter Henry Rolfs, s.n. - Campus Universitário,

36570-900 - Viçosa - Minas Gerais

Jonnathan de Souza Acre das Mercês - jtmerces@gmail.com

Universidade Federal de Viçosa

Av. Peter Henry Rolfs, s.n. - Campus Universitário,

36570-900 - Viçosa-Minas Gerais

Daniel Khede Dourado Villa-danielkdv@gmail.com

Universidade Federal de Viçosa

Av. Peter Henry Rolfs, s.n. - Campus Universitário,

36570-900 - Viçosa - Minas Gerais

Resumo: Durante o processo de formação em engenharia, é importante inserir, nas ações didático-pedagógicas, o uso de equipamentos e ferramentas que permitam uma aproximação com a vivência profissional. Este trabalho tem como objetivo desenvolver uma planta didática para o controle de velocidade e posição angular de um motor de corrente contínua, utilizando o método PID discretizado, importante estratégia de controle clássico. O objetivo dessa planta é fornecer as ferramentas práticas para o teste e a validação dos conceitos relacionados ao controle de processos. Os resultados obtidos asseguram sua relevância para a área de controle de processos e o potencial que o equipamento possui para auxiliar instituições de ensino superior na adequação de seus cursos às novas Diretrizes Curriculares Nacionais (DCNs) das engenharias.

Palavras-chave: Ensino. Didática. Engenharia. Controle de Processos. PID. 


\section{INTRODUÇÃO}

Criar sistemas capazes de melhorar a eficiência e a eficácia de processos industriais é um dos principais desafios do setor produtivo. Nesse cenário, as áreas de estudo relacionadas a Engenharia Elétrica e de Controle e Automação tornam-se protagonistas graças aos seus conteúdos curriculares baseados em disciplinas que abordam estratégias, métodos e equipamentos relacionados ao controle de processos.

Todavia, para que o protagonismo ocorra efetivamente, o engenheiro, desde a sua formação acadêmica, deve ter acesso a equipamentos e ferramentas que permitam experimentar situações reais. Essa é uma forma de assegurar a premissa que o Ministério da Educação (MEC) atualizou, em 2019, nas Diretrizes Curriculares Nacionais (DCNs) para os cursos de graduação em Engenharia.

Segundo o parecer da comissão do Conselho Nacional de Educação (2019), a revisão do texto busca "atender as demandas futuras por mais e melhores engenheiros". As DCNs dos cursos de graduação em engenharia definem os princípios, os fundamentos, as condições e as finalidades estabelecidas pela Câmara de Educação Superior do Conselho Nacional de Educação (CES/CNE) para aplicação, em âmbito nacional, na organização, no desenvolvimento e na avaliação dos cursos de graduação em Engenharia das Instituições de Educação Superior (IES).

Em comparação com a versão anterior do documento, de 2002, as novas DCNs de Engenharia trazem conceitos atuais como a formação baseada por competências. Ou seja, com foco na prática, através da aprendizagem ativa e de uma maior flexibilidade na constituição curricular. As atividades práticas, pouco mencionadas nas antigas versões das DCNs de Engenharia, são citadas nove vezes no documento atual. O número é apenas um indício da importância que o conhecimento prático ganhou.

Segundo a nova versão das DCNs, passam a ser obrigatórias as atividades de laboratório, já que, a partir delas, são desenvolvidas tanto competências gerais, quanto específicas, dado o enfoque de cada curso. As IESs são orientadas a estimular atividades que articulem teoria, prática e contexto de aplicação de maneira simultânea, o que se traduz no desenvolvimento das competências estabelecidas como perfil do egresso.

Diante do exposto, a proposta deste trabalho é desenvolver uma plataforma didática que proporcione um ambiente de aprendizagem experimental para disciplinas do curso de pósgraduação em Automação e Controle de Processos Agrícolas e Industriais da Universidade Federal de Viçosa (UFV). O equipamento permitirá o estudo de conceitos fundamentais de controle de processos, modelagem, sintonia de controladores, aquisição e tratamento de dados, microcontroladores, sistemas de supervisão e controle e eletrônica embarcada.

\section{ESPECIFICAÇÕES}

A planta didática realizará o controle de duas variáveis de processo de forma individual e não concomitante, sendo elas: 1- velocidade; e 2- posição angular de um motor de corrente contínua. A etapa de controle será realizada por um microcontrolador a partir de um controle discreto.

O sistema possibilitará o monitoramento e controle das variáveis através de modos de controle do tipo P, PI, PD, PID e On\Off. A operação ocorrerá em malha fechada, sendo que o sinal de feedback será gerado por um encoder incremental. A Figura 1 representa o diagrama em blocos da planta proposta. 
Figura 1 - Diagrama em Blocos.

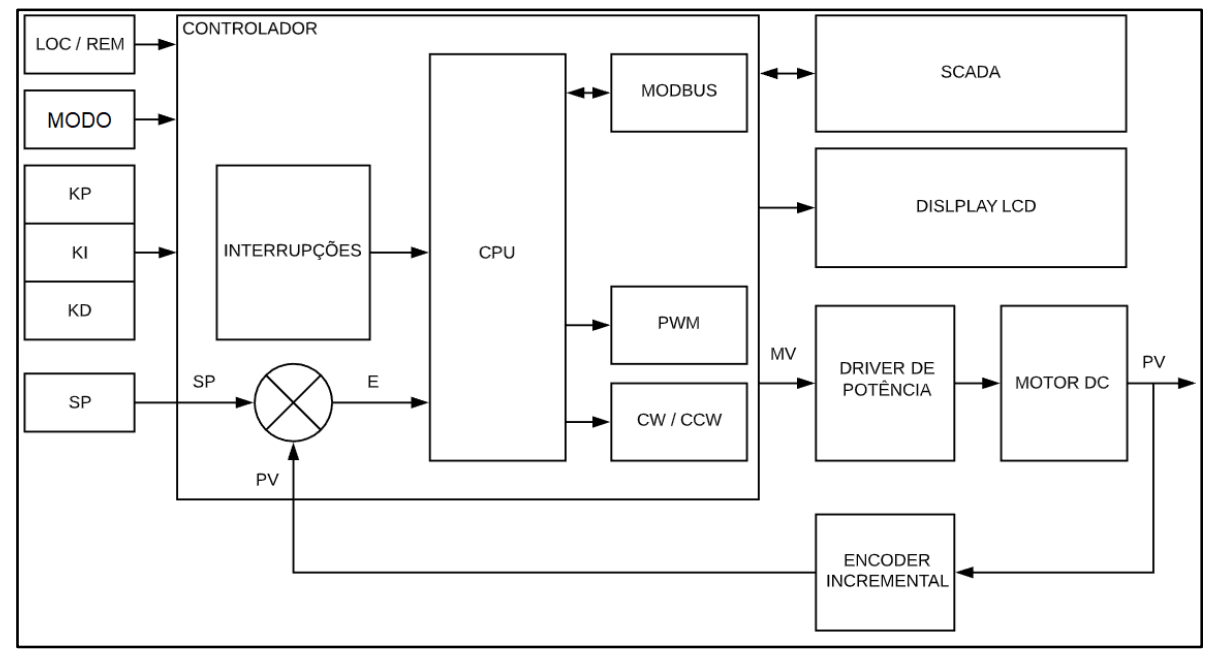

Fonte: Autor.

Toda a etapa de sintonia e operação poderá ser executada tanto em modo local, usando circuitos auxiliares da própria planta, bem como de forma remota, por software de supervisão e controle. A Figura 2 ilustra a disposição dos componentes na placa da planta didática.

Figura 2 - Planta Didática.

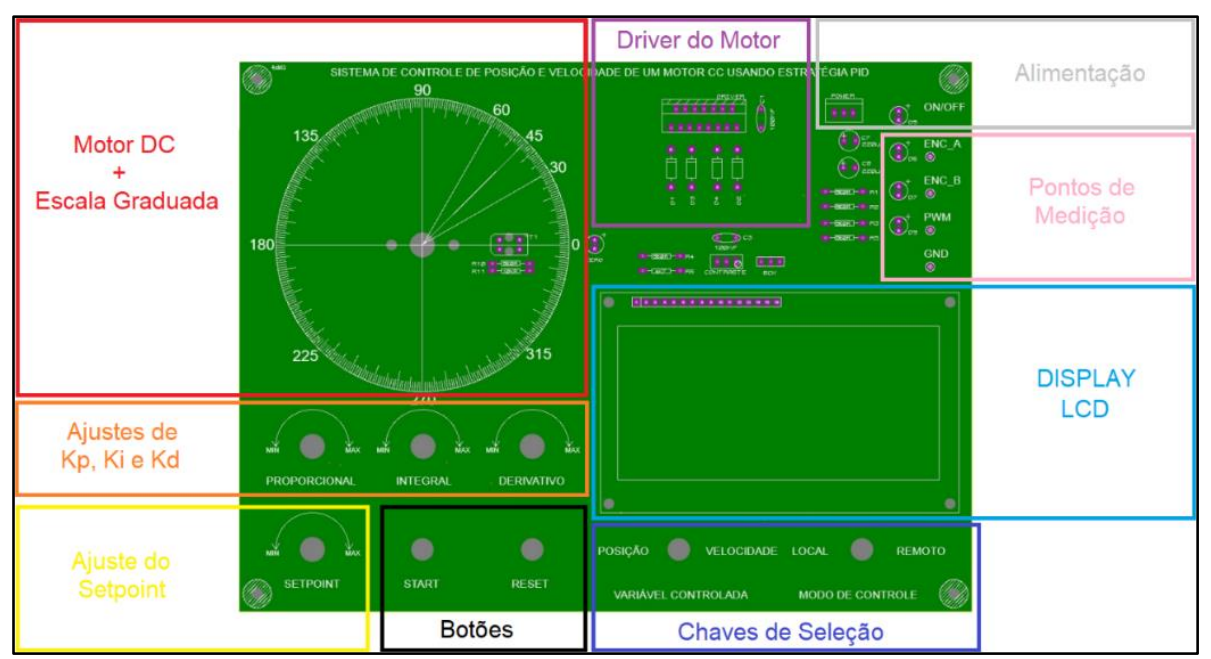

Fonte: Autor.

Dessa maneira, a planta será composta por motor DC e escala graduada, ajustes de Kp, $\mathrm{Ki}$ e Kd, ou seja, de proporcional, integral e derivativo, ajuste de setpoint, botões de start e reset, chaves de seleção de posição e de velocidade local, além de contar com o driver do motor, fonte de alimentação, pontos de medição e display LCD. Os próximos subtópicos detalharão as funções do atuador, do driver de potência, do controlador, da interface local e da interface remota.

\subsection{Atuador}

As variáveis de processo velocidade e posição angular serão geradas por um motor de corrente contínua. A Figura 3 apresenta o motor utilizado no projeto, com destaque para sua placa de encoder e sinais gerados quando o motor gira no sentido horário e anti-horário. 
Figura 3 - Motor DC com Encoder Incremental

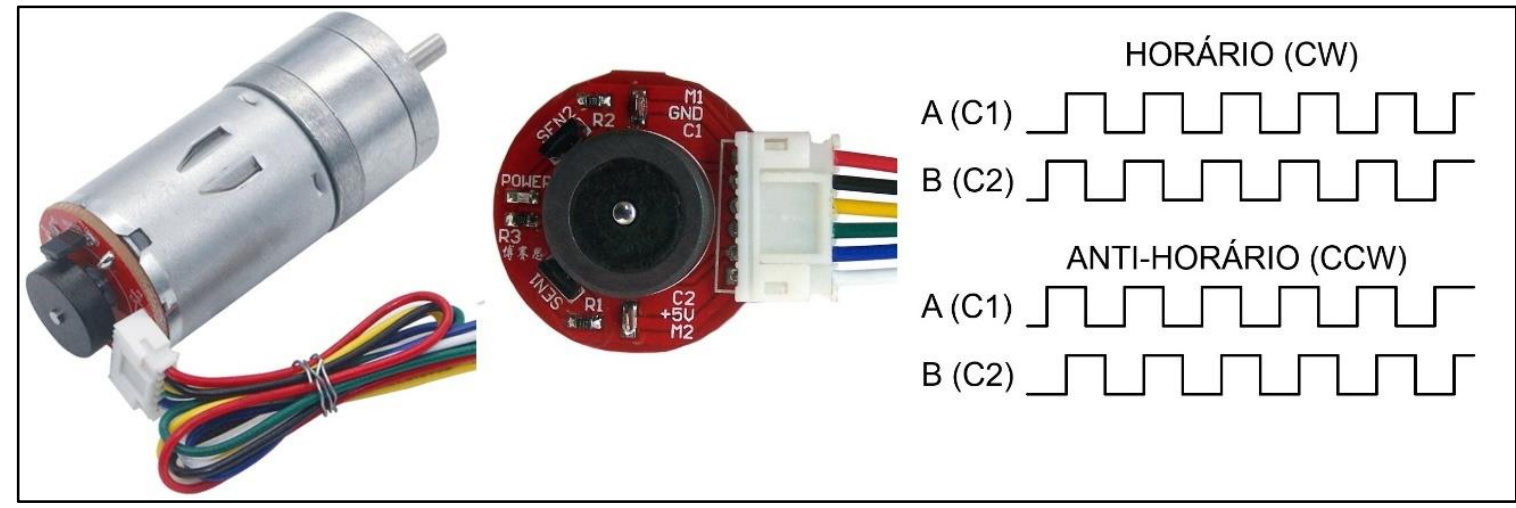

Fonte: Autor.

O encoder acoplado ao motor gera um sinal em quadratura, com uma defasagem de $90^{\circ}$. Dessa forma, é possível identificar não apenas a velocidade do motor, mas também o sentido de giro do seu eixo.

O eixo de trabalho do motor é acoplado ao principal por meio de uma caixa de redução mecânica. Assim, a velocidade do eixo principal é diferente do eixo de trabalho, sendo a relação entre as velocidades de 1 para 34,02. Como o encoder possui uma resolução de 11 pulsos por volta, o mesmo gerará 341,2 pulsos por volta do eixo de trabalho, sendo que esse valor é passado ao microcontrolador como parâmetro para que seja possível executar a lei de controle.

\subsection{Driver de Potência}

Para acionar o motor, utilizou-se o circuito integrado (CI) L298N, escolhido por possuir parâmetros elétricos compatíveis com o motor do projeto. A Figura 4 apresenta o CI e o diagrama elétrico do driver de potência.

Figura 4 - Driver de Potência.

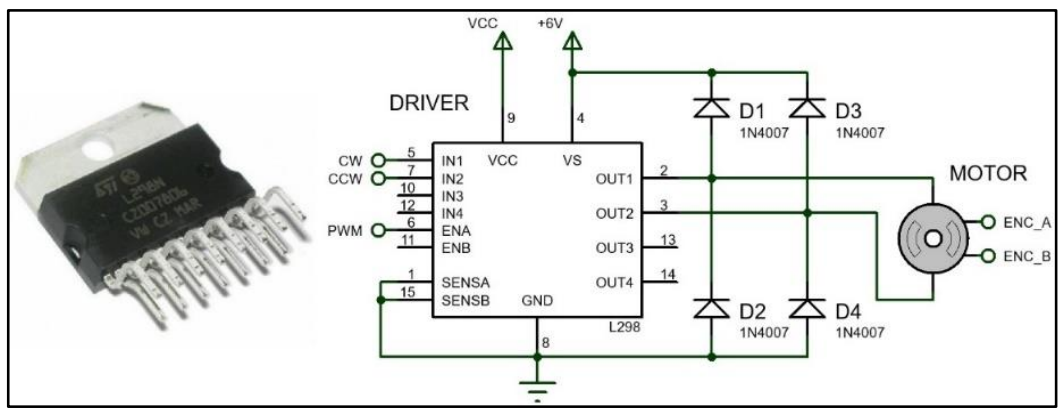

Fonte: Autor.

O controle do sentido de giro é feito através dos pinos IN1 (input 1) e IN2 (input 2) e a potência fornecida ao motor é controlada pelo pino ENA (Enable A), que está conectado a um pino do tipo PWM (Pulse Width Modulation).

\subsection{Controlador}

Para implementar a estratégia de controle, a quantidade de interrupções externas foi o fator determinante. Por isso, o microcontrolador escolhido foi o modelo ATMEGA 2560, da Atmel®, que apresenta seis interrupções externas, fundamentais para realizar a leitura dos sinais do encoder e dos potenciômetros digitais. Ademais, há uma quantidade significativa de informações na literatura acerca desse modelo, o que contribuiu para a escolha. 
A função do microcontrolador no projeto é implementar a lei de controle, além de estabelecer a leitura dos parâmetros, atualizar o valor das variáveis no display LCD e realizar a troca de informações com o sistema supervisório através do protocolo modbus RTU.

\subsection{Interface Local}

Quando o sistema opera em modo local, ou seja, quando todos os parâmetros são ajustados na própria placa da planta, os componentes, que fornecem os parâmetros de entrada para o microcontrolador, devem trabalhar em harmonia com o sistema supervisório. Esse passo é importante para que não haja divergências quando o modo de trabalho for alterado entre local e remoto.

Com isso, optou-se por utilizar encoders para a geração dos sinais, uma vez que, diferente dos potenciômetros analógicos, os encoders criam um sinal lógico através de um algoritmo de aquisição de dados, não referente ao seu posicionamento instantâneo.

\subsection{Interface Remota - Supervisório}

Segundo Constain (2011) o sistema de supervisão e controle emprega o uso de tecnologias computacionais e de comunicação para automatizar as etapas de monitoramento e controle de diferentes processos. Além disso, o sistema é responsável pela interação homem-máquina, porque permite que o usuário possa analisar e atuar de maneira direta em um processo produtivo (MARTINS, 2007).

Neste trabalho, a interface adquirirá variáveis de processo, tais como: setpoint (SP), variável controlada (PV), variável manipulada (MV), e apresentará esses valores de forma intuitiva, através de escalas e gráficos de tendência. Além disso, será possível realizar alterações na sintonia do controlador, modificando os valores dos ganhos proporcional, integral e derivativo, além de manipular o valor desejado (setpoint) quando a planta opera em modo remoto. A definição do modo de operação da planta é feita através de uma chave de seleção.

Ao abrir a aplicação de supervisão, a tela inicial poderá ser acessada, conforme ilustra a figura 5 :

Figura 5 - Tela Principal da Aplicação.

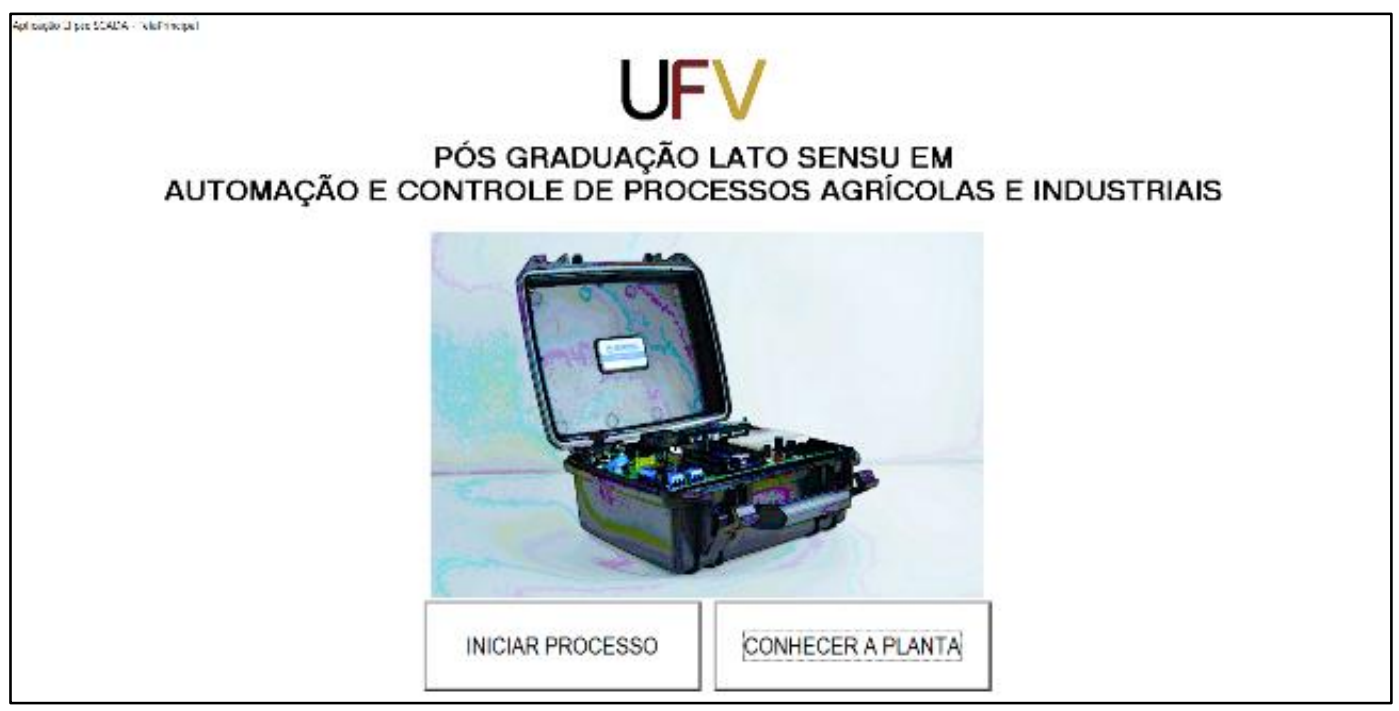

Fonte: Autor. 
Na tela inicial surgirá a planta energizada e conectada ao computador, no qual está funcionando a aplicação de supervisão. Dessa maneira, o usuário deverá escolher se iniciará o processo de controle através do botão "INICIAR PROCESSO" ou se desejará obter informações sobre a planta, através do botão "CONHECER A PLANTA".

Ao escolher a opção "INICIAR PROCESSO", o usuário será direcionado para uma das duas telas de operação: uma para o controle de velocidade e outra para o controle de posição angular. A tela que será aberta dependerá da posição de uma chave de seleção presente na planta didática, na qual o usuário escolhe a variável que deseja controlar. A depender da posição escolhida para a chave de seleção, apresenta-se uma tela de controle de processo que pode ser de velocidade ou de posição.

As duas telas possuem praticamente a mesma configuração, divergindo apenas nas escalas dos gráficos apresentados, já que a tela de controle de velocidade possui uma escala de -300 rpm a +300 rpm enquanto a de posição possui a escala variando entre $0^{\circ}$ e $400^{\circ}$. A Figura 6 ilustra a tela de controle de velocidade.

Figura 6 - Tela de Processo (Velocidade).

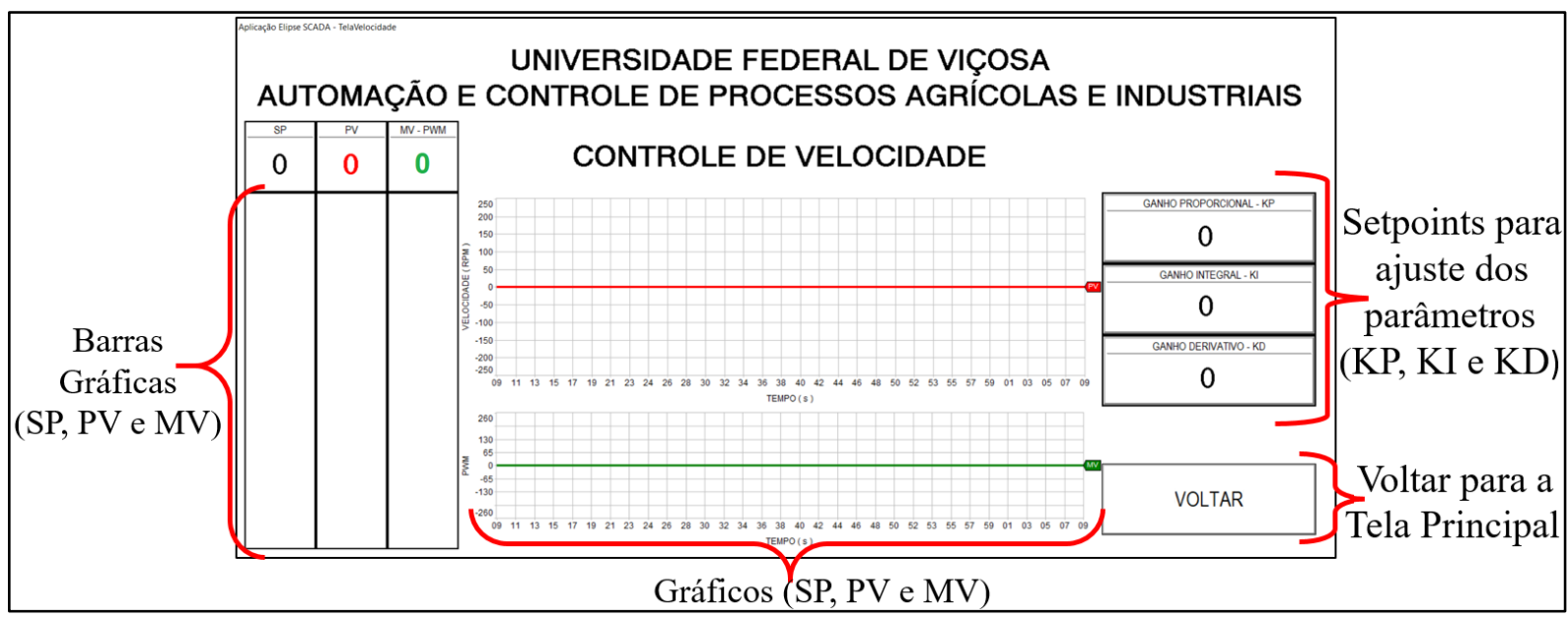

Fonte: Autor.

As telas de controle possuem dois gráficos: um que representa os valores de SP (Cinza) e PV (Vermelho) e outro que representa o valor de MV (Verde), que é um reflexo do PWM enviado ao driver de potência. Além disso, elas possuem mais dois displays que apresentam valores de PV e MV e quatro objetos de setpoint. Dessa forma, o usuário pode manipular os valores de SP, KP, KI e KD se o sistema estiver em modo remoto.

Caso na tela inicial seja escolhido a opção de conhecer a planta, o usuário será direcionado para uma tela na qual é apresentada a imagem da Figura 2, onde poderá obterá informações sobre os componentes da planta. 


\section{FIRMWARE}

O firmware implementado no microcontrolador tem sua lógica definida pelo fluxograma da Figura 7.

Figura 7 - Firmware do Microcontrolador

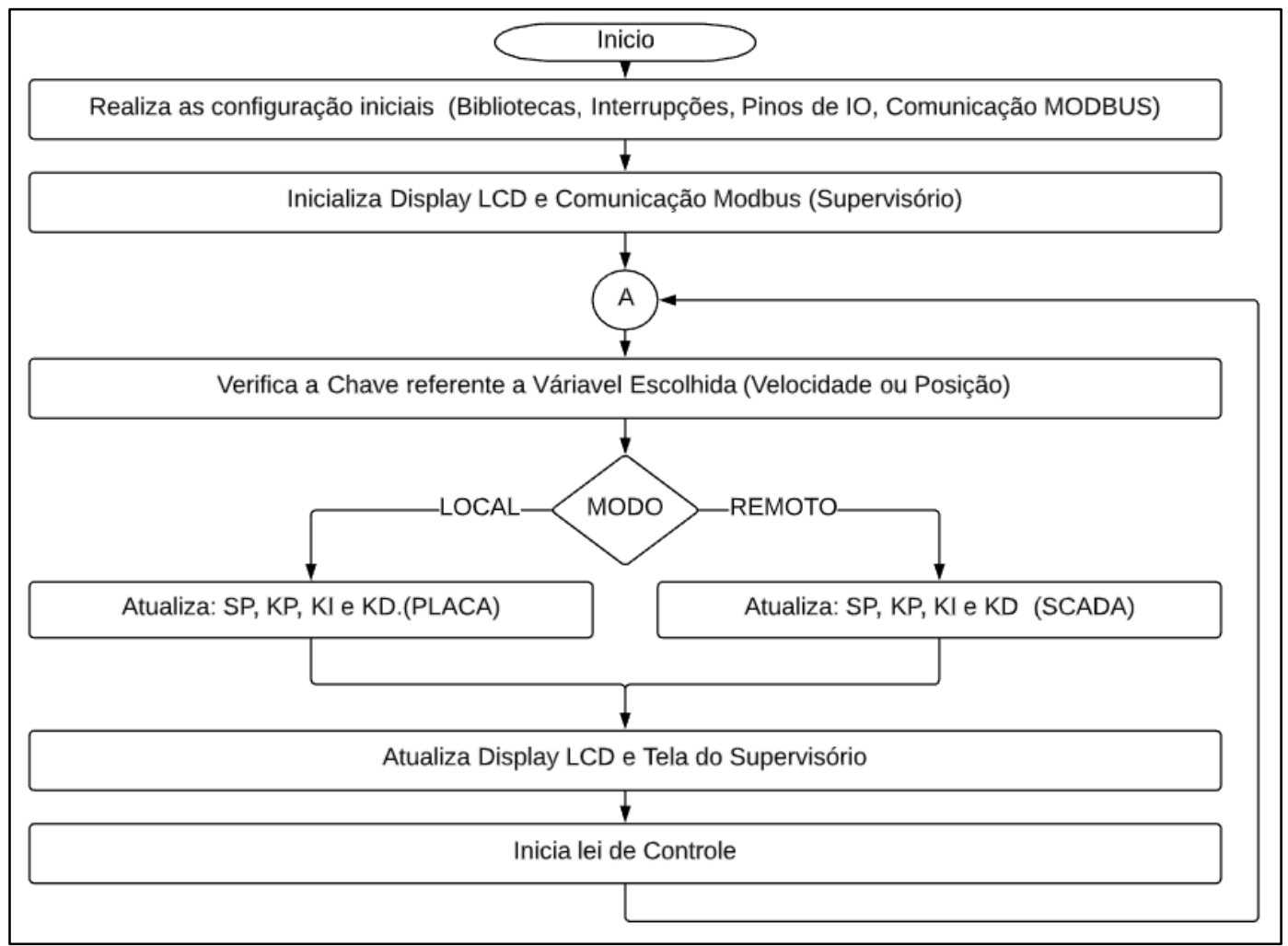

Fonte: Autor.

O algoritmo, após inicializar as configurações do microcontrolador; inicializar display e a comunicação com o supervisório, verifica a posição da chave de seleção de variável (velocidade ou posição). Em seguida, verifica se irá operar em modo local ou remoto.

A escolha do "modo local" possibilitará a leitura dos parâmetros enviados pelos periféricos presentes na planta e, por consequência, a aplicação da lei de controle. Na opção remoto os parâmetros serão recebidos via supervisório. Em ambos os modos há o envio de valores aos supervisórios para gerar os gráficos de tendência.

\section{RESULTADOS}

O objetivo deste trabalho não foi analisar métodos de sintonia de controladores, tampouco avaliar modos de controle. Ele visou desenvolver uma ferramenta que permita que as análises supracitadas possam ser realizadas, tendo como objeto de estudo o PID discretizado em um controlador de variáveis de processo de um motor de corrente contínua. Portanto, os tópicos deste capítulo se referem aos resultados encontrados a respeito da funcionalidade quando se utilizou a planta, e não sobre o resultado do controle. 


\subsection{Controle de Velocidade}

Para avaliar o desempenho do projeto no controle de velocidade, o equipamento foi configurado para atuar em modo remoto. Com isso, o valor da variável desejada (SP) foi alterado entre degraus positivos e negativos a cada intervalo de aproximadamente cinco segundos. Os resultados são apresentados na Tabela 1.

Tabela 1 - Parâmetros utilizados no teste de controle de velocidade.

\begin{tabular}{cc}
\hline Período (segundos) & Setpoint $(\mathrm{rpm})$ \\
\hline 0 & 100 \\
5 & 200 \\
10 & 50 \\
15 & 0 \\
20 & -100 \\
25 & -200 \\
30 & -50 \\
35 & 0 \\
\hline
\end{tabular}

Fonte: Autor.

A Figura 8 ilustra o comportamento das variáveis de processo durante as diversas alterações do Setpoint, sendo que na cor verde da figura é possível acompanhar a variável manipulada. No caso retratado ela representa a saída PWM que será enviada ao driver de potência.

Figura 8 - Gráfico de Controle de Velocidade.

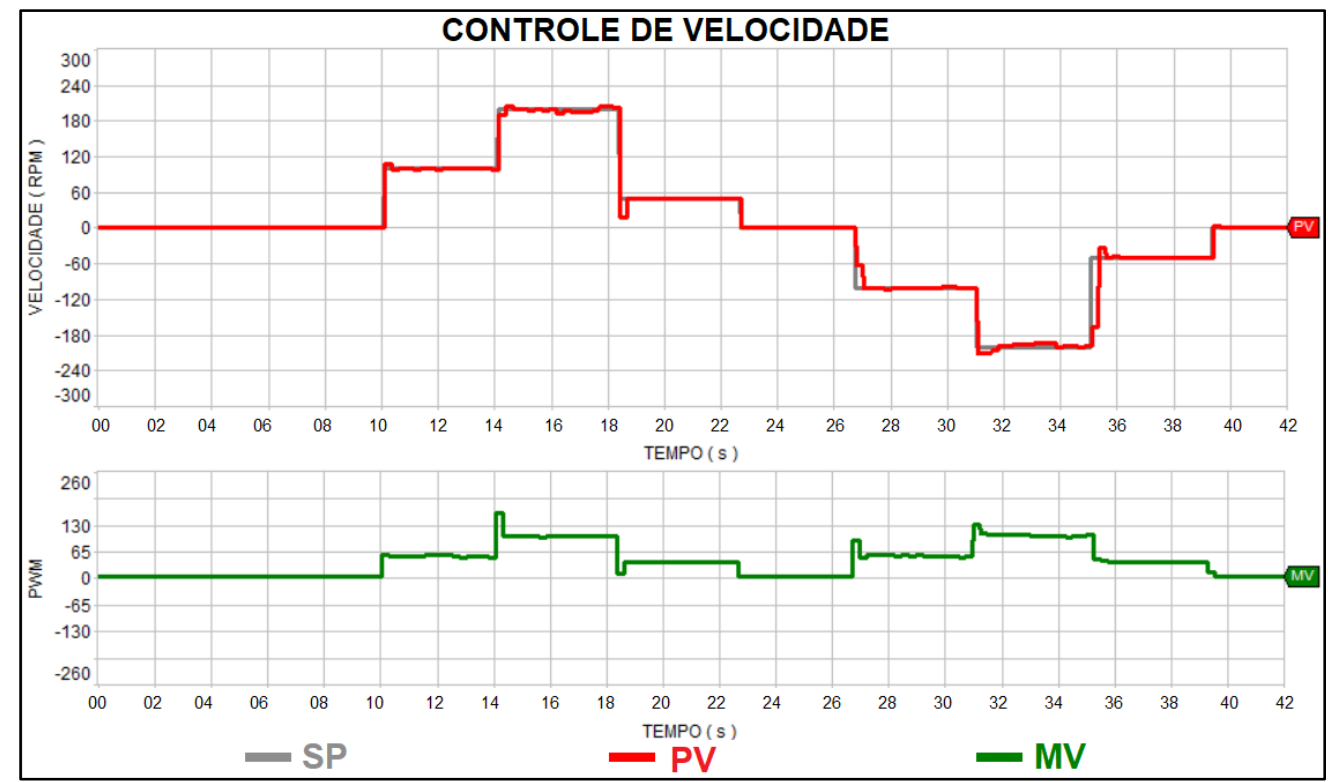

Fonte: Autor.

Um fato que merece destaque é que a variável manipulada sempre apresenta um valor positivo, mesmo em situações que o sentido de giro é alterado. O PWM interfere apenas na potência entregue ao motor, e consequentemente em sua velocidade. O sentido de giro é selecionado por pinos de controle, descritos anteriormente na seção sobre o driver de potência. 


\subsection{Controle de Posição}

Para avaliar o desempenho do projeto no controle de velocidade, o equipamento foi configurado para atuar em modo remoto, sendo que o valor da variável desejada (SP) foi alterado conforme a Tabela 2 .

Tabela 2 - Parâmetros utilizados no teste de controle de posição angular.

\begin{tabular}{cc}
\hline Período (segundos) & Setpoint (ângulo) \\
\hline $3<$ & $0^{\circ}$ \\
$3-13$ & $180^{\circ}$ \\
$13-23$ & $0^{\circ}$ \\
$23-33$ & $90^{\circ}$ \\
$33-43$ & $180^{\circ}$ \\
$43-53$ & $60^{\circ}$ \\
$>53$ & $0^{\circ}$ \\
\hline \multicolumn{2}{c}{ Fonte: Autor. }
\end{tabular}

A Figura 9 ilustra o comportamento das variáveis de processo durante as diversas alterações do setpoint. A linha cinza representa o SP e a linha vermelha o PV, sendo que ambos são apresentados em rotações por minuto (rpm).

Figura 9 - Gráfico de Controle de Posição Angular.

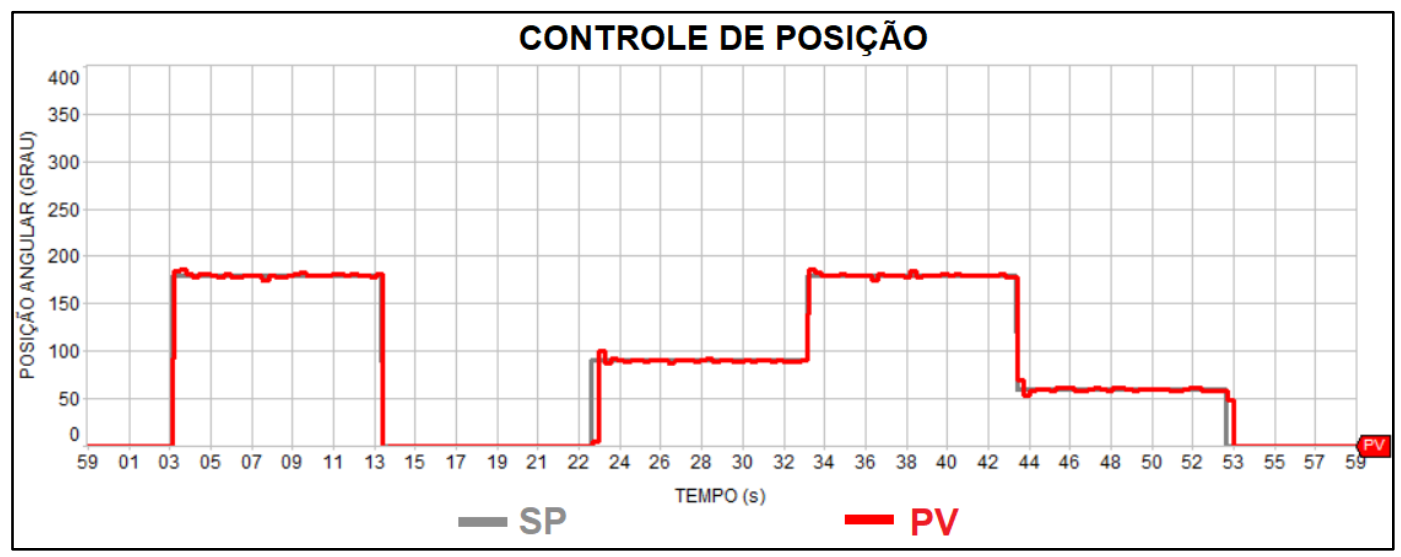

Fonte: Autor.

\section{CONSIDERAÇÕES FINAIS}

Os resultados alcançados nos testes da planta foram satisfatórios, comprovando a aplicabilidade do projeto em disciplinas relacionadas ao controle de processos. Sendo assim, o projeto apresenta as características adequadas, do ponto de vista técnico, para compor infraestruturas laboratoriais de instituições de ensino superior que ofertam cursos de graduação em engenharia.

Ademais, a planta didática se configura como uma ferramenta que auxilia o aprendizado prático, o que aproxima os projetos pedagógicos das instituições às novas diretrizes curriculares nacionais para os cursos de engenharia.

O funcionamento completo da planta pode ser observado através do link: https://www.youtube.com/watch?v=T9fkZAQ_JpM\&t 


\section{REFERÊNCIAS}

ABMES - Associação Brasileira de Mantenedoras do Ensino Superior. Resolução no 2 de 24 de abril de 2019. Diretrizes Curriculares Nacionais do Curso de Graduação em Engenharia.

Diário Oficial da união: seção 1, nº 80, p. 43, 26 abr. 2019. Disponível em:

<https://abmes.org.br/arquivos/legislacoes/Resolucao-CNE-CES-002-2019-04-24.pdf > .

Acesso em: 31 mai. 2020.

CONSELHO NACIONAL DE EDUCAÇÃO. Parecer $n^{\circ}$ 1/2019 de 23 jan. 2020. Diretrizes Curriculares Nacionais do Curso de Graduação em Engenharia. Diário Oficial da União: parte 1, seção 1, p. 19, 23 abr. 2019. Disponível em: 〈http://portal.mec.gov.br/docman/janeiro-2019pdf/106581-pces001-19/file >. Acesso em: 30 mai. 2020.

CONSTAIN, N. B. P. Integração de sistemas SCADA com a implementação de controle supervisório em CLP para sistemas de manufatura. 2011, 143p. Dissertação (Mestrado em Engenharia de Automação e Sistemas) - Curso de Pós-graduação em Engenharia de Automação e Sistemas, Universidade Federal de Santa Catarina - Florianópolis, SC, 2011.

MARTINS, G. M. Princípios de automação industrial. Notas de aula. Universidade Federal de Santa Maria, 2007.

SOUZA, P. F. R. Sistema de Controle de Posição e Velocidade de Um Motor CC. [S. 1], 1 vídeo (6m). Disponível em: https://www.youtube.com/watch?v=T9fkZAQ_JpM\&t. Acesso em: 7 ago. 2020.

VILLA, D. K. D. Automação de um sistema condicionador de ar aplicado a processos de secagem e armazenagem de produtos agrícolas. 2017. 10p. Dissertação (Mestrado em Engenharia Agrícola) - Curso de Pós-graduação em Engenharia Agrícola, Universidade Federal de Viçosa - Viçosa, MG, 2017.

\section{DIDACTIC PLANT FOR SPEED AND ANGULAR POSITION CONTROL OF A CONTINUOUS CURRENT MOTOR: USE OF THE DISCRETIZED PID METHOD}

Abstract: During the engineering training process, it is important to insert, in the didacticpedagogical actions, the use of equipment and tools that allow an approximation with the professional experience. This work aims to develop a didactic plan for controlling the speed and angular position of a direct current motor, using the discretized PID method, an important classic control strategy. The purpose of this plant is to provide practical tools for testing and validating concepts related to process control. The results obtained ensure its relevance to the area of process control and the potential that the equipment has to assist higher education institutions in adapting their courses to the new National Curriculum Guidelines (DCNs) of engineering.

Key-words: Teaching. Didatics. Engineering. Process control. PID. 